\title{
Loss of memory B cells during chronic HIV infection is driven by Foxo3a- and TRAIL-mediated apoptosis
}

\author{
Julien van Grevenynghe, ${ }^{1,2,3,4}$ Rafael A. Cubas, ${ }^{4}$ Alessandra Noto, ${ }^{1,2,3,4}$ \\ Sandrina DaFonseca,1,2,3,4 Zhong He,1,2,3,4 Yoav Peretz,1,2,3 Abdelali Filali-Mouhim,1,2,3,4 \\ Franck P. Dupuy,1,2,3,4 Francesco A. Procopio, 1,2,3,4 Nicolas Chomont, 1,2,3,4 Robert S. Balderas, ${ }^{5}$ \\ Elias A. Said, ${ }^{6}$ Mohamed-Rachid Boulassel,7 Cecile L. Tremblay,1,2 Jean-Pierre Routy, ${ }^{3,7}$ \\ Rafick-Pierre Sékaly, 1,2,3,4,8 and Elias K. Haddad'1,2,3,4,8
}

\begin{abstract}
${ }^{1}$ Vaccine and Gene Therapy Institute — Florida, Port St. Lucie, Florida, USA. ${ }^{2}$ Centre de recherche du Centre hospitalier de l'Université de Montréal (CRCHUM), Hôpital Saint-Luc, Québec, Canada. 'Laboratoire d'Immunologie, Département de Microbiologie et d'Immunologie, Université de Montréal, Montréal, Québec, Canada. ${ }^{4}$ Institut National de la Santé et de la Recherche Médicale U743, CRCHUM, Université de Montréal, Montréal, Québec, Canada. ${ }^{5} \mathrm{BD}$ Biosciences, San Diego, California, USA. ${ }^{6}$ Department of Microbiology and Immunology, College of Medicine, Sultan Qaboos University, Muscat, Oman. ${ }^{7}$ Immunodeficiency Service and Division of Haematology, Royal Victoria Hospital, McGill University Health Center, McGill University, Montréal, Québec, Canada. ${ }^{8}$ Department of Microbiology and Immunology, McGill University, Montréal, Québec, Canada.
\end{abstract}

\begin{abstract}
Loss of memory B cells occurs from the onset of HIV-1 infection and persists into the chronic stages of infection. Lack of survival of these cells, even in subjects being treated, could primarily be the consequence of an altered local microenvironment induced by HIV infection. In this study we showed that memory B cell survival was significantly decreased in aviremic successfully treated (ST) subjects compared with subjects who control viral load as a result of natural immunity (elite controller [EC]) or with uninfected control (HIV-) subjects. The lower survival levels observed in memory B cells from ST subjects were the result of disrupted IL-2 signaling that led to increased transcriptional activity of Foxo3a and increased expression of its proapoptotic target TRAIL. Notably, memory B cell survival in ST subjects was significantly enhanced by the addition of exogenous IL-2 in a Foxo3a-dependent manner. We further showed that Foxo3a silencing by siRNA resulted in decreased expression of TRAIL and apoptosis levels in memory B cells from ST subjects. Our results thus establish a direct role for Foxo3a/TRAIL signaling in the persistence of memory $B$ cells and provide a mechanism for the reduced survival of memory $B$ cells during HIV infection. This knowledge could be exploited for the development of therapeutic and preventative HIV vaccines.
\end{abstract}

\section{Introduction}

In addition to progressive $\mathrm{T}$ cell dysfunction and cell death, HIV infection itself leads to early and profound deregulation of B cell physiology, homeostasis, and function. These are manifested by polyclonal activation of undifferentiated naive B cells (1), induction of hypergammaglobulinemia (2), increased expression of activation markers (3), high frequencies of apoptotic cells (4), poor responsiveness to antigenic and mitogenic stimulation $(5,6)$, and a progressive depletion of peripheral CD2 $7^{+}$memory B cells (7). Of note, this loss of memory B cells already occurs from the onset of acute infection $(8,9)$. Interestingly, successfully treated (ST) subjects, with drug-suppressed viremia, still show low frequencies of $\mathrm{CD} 27^{+}$memory B cells and low production of Abs that are not fully restored by highly active antiretroviral treatments (HAARTs) (10-13). On the other hand, elite controller (EC) subjects, who naturally control viral replication and maintain $\mathrm{CD}^{+} \mathrm{T}$ cell counts comparable to those of uninfected control $\left(\mathrm{HIV}^{-}\right)$subjects in the absence of HAART, show no memory $\mathrm{B}$ cell loss and display broad and functional $\mathrm{T}$ and B cell memory responses (13-16). ST subjects thus provide ideal subjects to identify defects in memory B cell survival, whereas

Authorship note: Rafick-Pierre Sékaly and Elias K. Haddad are co-senior authors. Conflict of interest: The authors have declared that no conflict of interest exists. Citation for this article: J Clin Invest. 2011;121(10):3877-3888. doi:10.1172/JCI59211. studying memory B cells in EC subjects could lead to the identification of unique characteristics of memory B cell survival associated with natural control of HIV infection.

We previously identified the Foxo3a pathway as a major regulator of central memory $\mathrm{T}$ cell survival $(15,17)$. Foxo3a is a transcription factor that is constitutively expressed in hematopoietic cells and can induce the transcription of proapoptotic target genes such as Bim, FasL, and TRAIL (18-20). Phosphorylation of Foxo3a by several external signals including $\gamma$-chain receptor cytokines such as IL- 2 or T and B cell receptors $(17,21,22)$ leads to its degradation in the cytoplasm and inhibition of its transcriptional activity $(18,23,24)$. Evidence in the literature suggests that Foxo3a might participate in normal B cell development and homeostasis. For example, Foxo3a has been shown to be involved in B cell differentiation, where it can link BcR signaling with recombination machinery and affect VDJ recombination and affinity maturation $(25,26)$. In that context, Foxo3a-deficient mice show reduced numbers of pre-B cells and recirculating B cells when compared with wild-type counterparts (27).

Although transcription factors such as Bcl-6 and Mcl-1 have been previously shown to play critical roles in the generation of memory B cells $(28,29)$, little is known about the implication of Foxo3a in the survival or the development of memory B cells in the context of acute and chronic viral or even bacterial infections. In this study, we investigated the molecular mechanisms responsible for the lack 
A

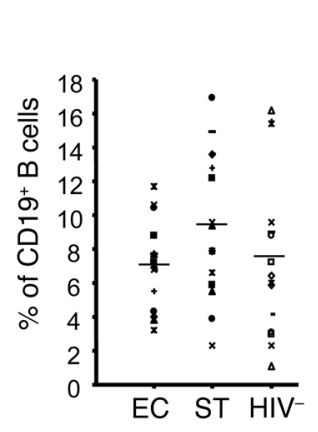

$$
\text { D }
$$

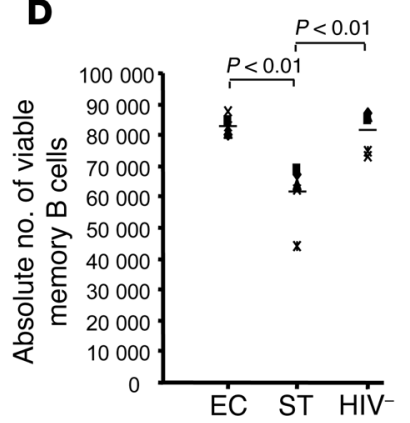

B

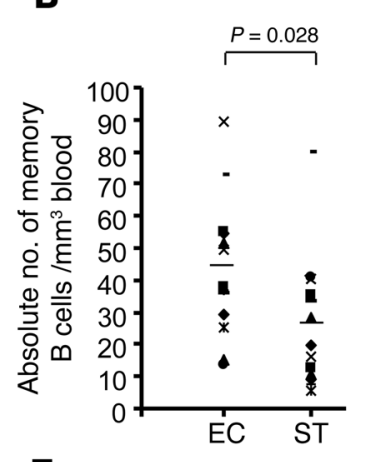

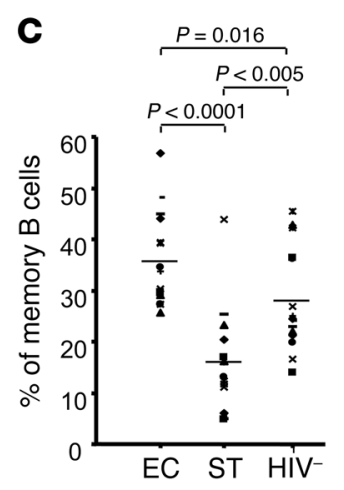
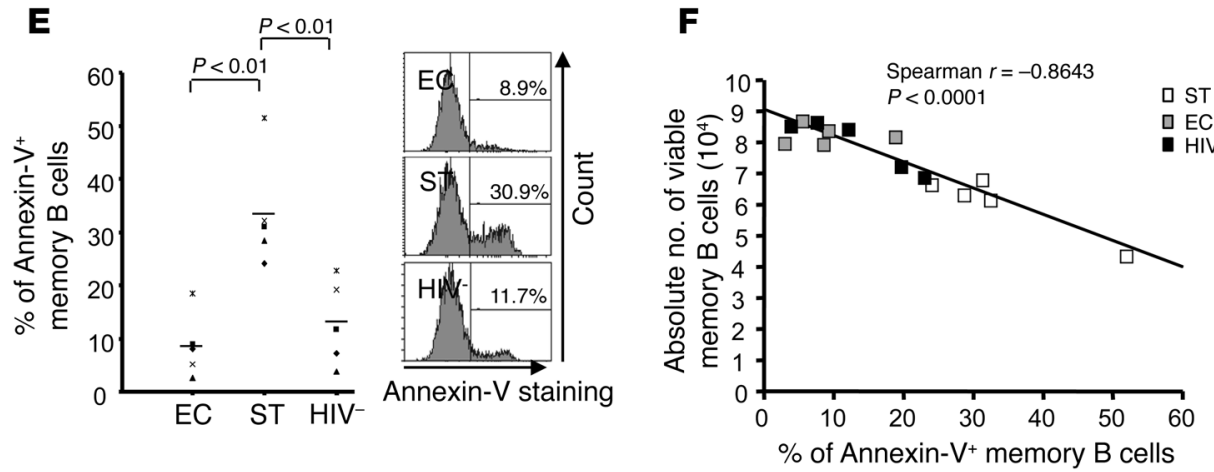

\section{Figure 1}

Loss of peripheral CD27+ memory B cells in ST subjects is associated with higher levels of apoptosis. (A-C) Ex vivo PBMCs from ST, EC, and HIV- $^{-}$subjects were stained with anti-CD3-PB (PB, pacific blue), anti-CD19-Alexa Fluor 700, and anti-CD27-APC Cy7 Abs to measure (A) the percentages of CD19+ $B$ cells within total lymphocytes, (B) the absolute number of CD27+ memory B cells per $\mathrm{mm}^{3}$ of blood in EC and ST subjects using available clinical data for $\mathrm{HIV}^{+}$subjects $(n=13)$, and $(\mathbf{C})$ the frequency of the memory population in total $\mathrm{B}$ cells $(n=15)$. (D) Memory $\mathrm{B}$ cells $\left(10^{5}\right)$ from $\mathrm{HIV}$ and $\mathrm{HIV}^{-}$subjects were purified and cocultured for 7 days with $9 \times 10^{5}$ autologous CD19-depleted PBMCs in the presence of $10 \mu \mathrm{M}$ AZT. The absolute number of viable memory B cells was determined on day 7 using trypan blue exclusion $(n=5)$. (E) The percentages of apoptotic memory B cells from ST, EC, and HIV- subjects on day 7 were analyzed using annexin V-APC staining. The histograms are representative of raw data from 5 independent experiments. $(F)$ The correlation between the absolute numbers of viable memory B cells and the frequency of apoptosis of this population at day 7 of coculture was calculated ( $n=15$; Spearman test).

of memory B cell survival in chronically HIV-infected subjects (who displayed a decrease in the frequency of memory B cells) and in EC and HIV- subjects (who maintained a statistically significant higher numbers of memory B cells). Our results identified a critical role for the Foxo3a/TRAIL pathway in memory B cell survival.

\section{Results}

$C D 27^{+}$memory B cells from ST subjects are short lived and apoptotic. Previous reports have indicated that $\mathrm{HIV}^{+}$patients, including those undergoing HAART, show significantly lower frequencies of memory B cells when compared with uninfected donors (10-13). To better understand the underlying mechanisms responsible for the decrease in the numbers of memory B cells, we measured the frequencies and absolute numbers of peripheral memory $B$ cells in chronically HIV-infected subjects as well as in uninfected donors (HIV-). Supplemental Table 1 (supplemental material available online with this article; doi:10.1172/JCI59211DS1) summarizes the clinical and virological data of the 2 groups of aviremic chronically HIV-infected (EC and ST) subjects. We first compared the ex vivo frequencies and absolute numbers of total peripheral B cells in ST, $\mathrm{EC}$, and HIV- subjects. Total B cells (defined as $\mathrm{CD}^{-}{ }^{-} \mathrm{CD} 19^{+}$) were similar in their frequencies and absolute numbers in all groups tested (Figure 1A and Supplemental Table 1). In contrast, the absolute numbers $(P=0.028 ; n=13)$ (Figure 1B) and frequencies (Figure 1C) of CD27 $7^{+}$memory B cells from ST subjects $(16.2 \% \pm 9.8)$ were significantly lower than those from EC subjects $(35.9 \% \pm 9.2 \% ; P<0.0001$; $n=15)$ or HIV- subjects $(28 \% \pm 10 \% ; P<0.005 ; n=15)$. Interestingly, EC subjects also displayed significantly higher frequencies of memory B cells when compared with HIV- subjects $(P=0.016 ; n=15)$. Total CD2 $7^{+}$memory B cells can be divided into 4 distinct memory subsets based on IgM and IgD expression (30-32) (Supplemental Figure 1A). Our results showed that the decreased frequencies of memory B cells in ST subjects occurred in all memory B cell subsets when compared with EC subjects, and in $\mathrm{IgD}^{-}$subsets when compared with HIV- subjects (Supplemental Figure 1B; $n=15$ ).

We next determined whether the lower frequencies of memory $\mathrm{B}$ cells in ST subjects were the consequence of increased levels of apoptosis. Survival levels of resting memory B cells from ST, EC, and HIVsubjects were thus assessed in a 7-day coculture assay in the presence of autologous B cell-depleted PBMCs. The latter might provide infection-associated extrinsic factors that could influence memory $B$ cell survival. Using this in vitro system, we observed that by day 7 the absolute numbers of viable memory B cells from ST subjects were significantly lower than those from EC and HIV- $^{-}$subjects $(P<0.01$; $n=5$ ) (Figure 1D). Interestingly, the reduced numbers of viable memory B cells in ST subjects at day 7 were the direct consequence of higher levels of apoptosis $(33 \% \pm 10.6 \%)$ when compared with EC $(9 \% \pm 6 \% ; P<0.01 ; n=5)$ and HIV- subjects $(13 \% \pm 8 \% ; P<0.01$; 
A

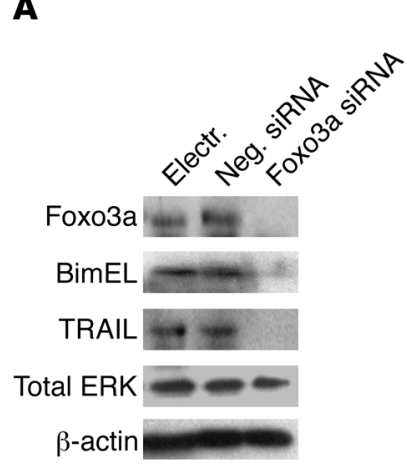

B

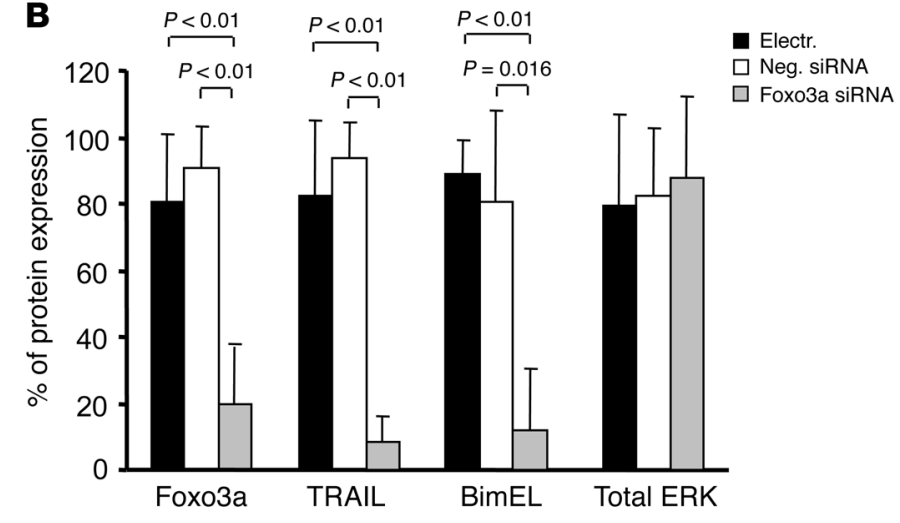

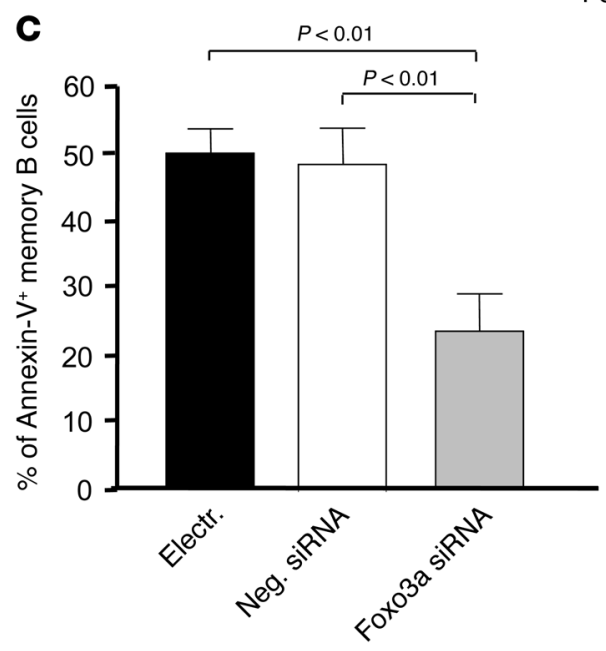

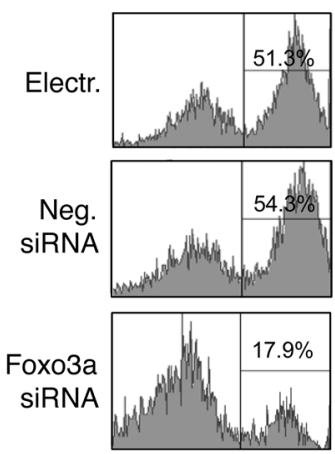

Figure 2

Foxo3a regulated the premature death of memory B cells in ST subjects. Purified memory B cells from ST subjects were either electroporated (electr.), or transfected with negative (neg.) siRNAs or with specific Foxo3a siRNAs, and then cocultured for 7 days with autologous CD19- PBMCs. (A) The efficacy of silencing Foxo3a using siRNAs was determined on purified memory B cells from ST subjects after 2 days of coculture using Western blot analysis. No changes were observed in the levels of total ERK expression, confirming the specificity of Foxo3a silencing. (B) Densitometric quantification of specific bands was performed using ImageQuant software $(15,17)$. The levels of expression of each protein were normalized to actin and were later expressed as the ratio of densitometric values of protein of interest divided by densitometric values of actin within the same blot. Results shown represent the mean relative expression \pm SD of 5 independent experiments. (C) The levels of apoptosis in the presence or absence of Foxo3a were assessed on transfected memory B cells from ST subjects on day 7 by annexin V-APC staining (mean \pm SD). The histograms shown are representative of raw data from 5 independent experiments.

$n=5)$ (Figure 1E). This was further confirmed by the highly statistically inverse correlation (Spearman $r=-0.8643 ; P<0.0001$; $n=15)$ between the absolute numbers of viable memory $\mathrm{B}$ cells and levels of apoptosis at day 7 of coculture within all studied subjects (Figure 1F). The increased levels of apoptosis were not due to higher levels of activation as memory B cells from all these subject groups showed comparable frequencies [\% of positive cells] and expression levels [MFI] of memory B cell activation and proliferation markers including HLA-DR, CD40, CD80, CD86, and Ki67 at the onset of the coculture (Supplemental Figure 2, A-F). Of note, a significant increase in the frequency of apoptotic memory B cells from ST subjects compared with HIV- subjects was already apparent by day 3 of coculture, as measured by annexin V staining (Supplemental Figure $3 \mathrm{~A})$. Accordingly, the absolute numbers of viable memory B cells from ST subjects were significantly lower than those from HIV- subjects at day 7 (Supplemental Figure 3B).

Overall these results demonstrated that peripheral memory B cells from ST subjects showed increased levels of apoptosis and reduced capacity to survive in the 7-day coculture assay when compared with EC and HIV $^{-}$subjects.

Foxo3a protects ST memory $B$ cells from apoptosis. Given the key role that the Foxo3a transcription factor plays in regulating memory $\mathrm{T}$ cell survival during HIV infection (15), we determined whether it was involved in mediating the premature memory B cell death observed in ST subjects (Figure 1, D and E). As Foxo3a could induce transcription of the proapoptotic molecules TRAIL, Bim, and others, we thus determined whether directly interfering with Foxo3a expression using siRNA silencing could protect memory B cells in ST subjects from apoptosis and could enhance their survival in the 7-day coculture assay. To this aim, purified memory B cells from ST subjects were either electroporated or transfected with siRNAs specific for Foxo3a or with scrambled siRNAs (negative control) and then cocultured with autologous CD19depleted PBMCs. Memory B cells transfected with Foxo3a siRNAs (purified at day 2 of coculture) exhibited an average of $71 \%$ reduction in Foxo3a expression using densitometric analysis $(P<0.01 ; n=5)$ (Figure 2, A and B). Accordingly, Foxo3a proapoptotic targets including BimEL and TRAIL were also significantly downregulated following Foxo3a silencing (an average of $68.4 \%[P=0.016]$ and $85.2 \%[P<0.01]$, respectively; $n=5)$ (Figure 2 , A and B).

We then assessed, using annexin $\mathrm{V}$ staining, the survival of memory B cells at day 7 in the presence or absence of Foxo3a. Our results showed that treatment of memory B cells from ST subjects with Foxo3a siRNAs led to a significant improvement in memory B cell survival when compared with negative siRNA-transfected cells $(48.9 \% \pm 12 \%$ and $23.6 \% \pm 12.5 \%$ of apoptosis for negative siRNAand Foxo3a siRNA-transfected cells, respectively; $P<0.01 ; n=5$ ) (Figure 2C). Interestingly, silencing Foxo3a within memory B cells from ST subjects resulted in reduced levels of apoptosis similar to those observed in coculture of transfected memory B cells from EC subjects (19.9\% $\pm 12.2 \%$ of apoptosis) (data not shown). Thus, 
A
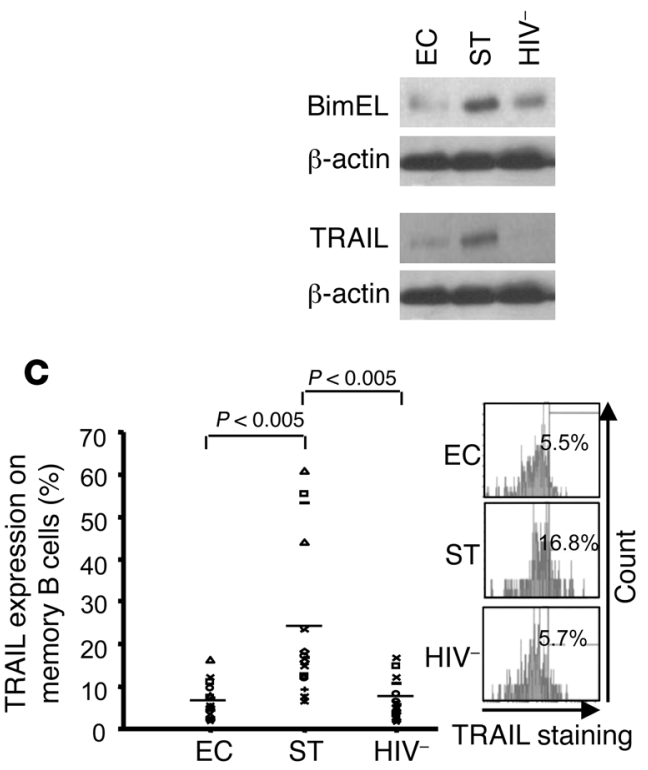

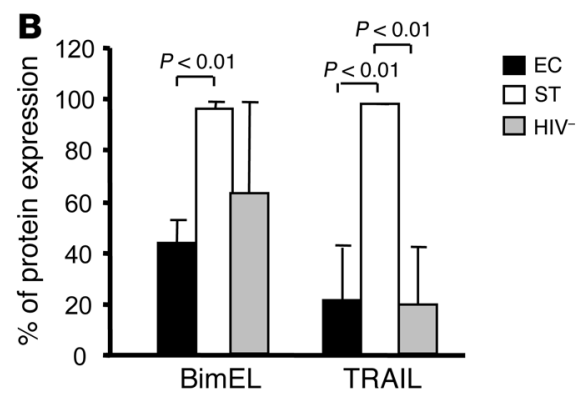

D

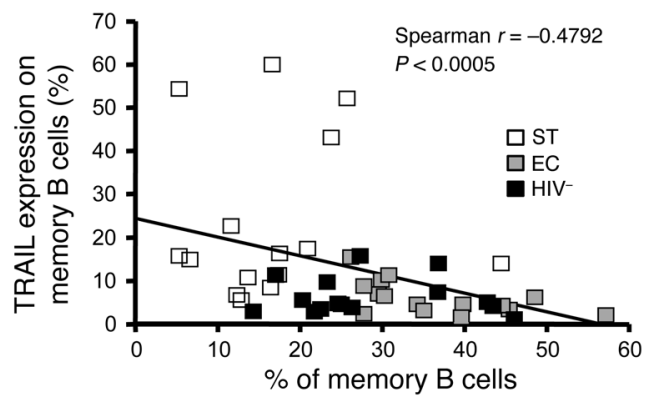

E

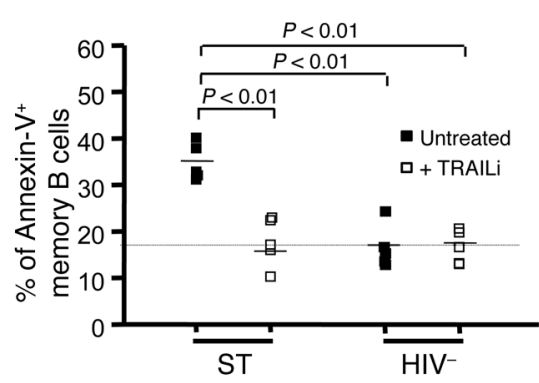

$\mathbf{F}$

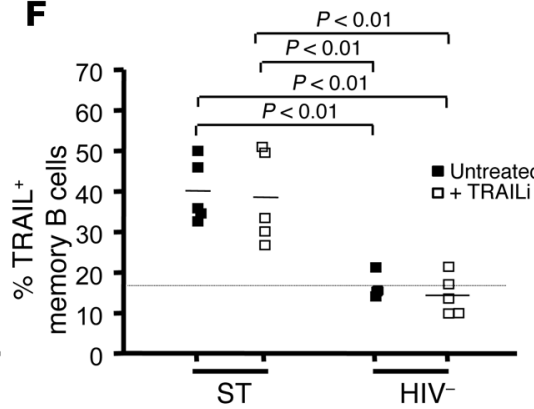

G

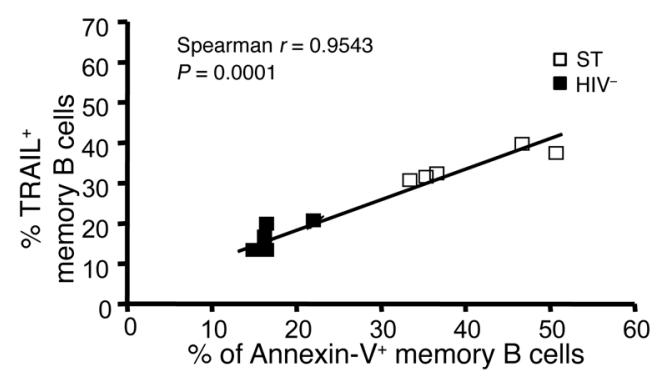

Figure 3

Interfering with TRAIL pathway enhances the survival of memory B cells from ST subjects. (A) Western blot analysis of Bim and TRAIL expression was performed on ex vivo cell lysates of highly purified memory B cells isolated from $\mathrm{HIV}^{+}$and $\mathrm{HIV}^{-}$subjects. (B) Densitometric quantification of 5 independent experiments was performed using ImageQuant software (mean \pm SD). (C) Ex vivo PBMCs from HIV+ and HIV- subjects were stained with 7AAD, anti-CD3-PB, anti-CD19-Alexa Fluor 700, anti-CD27-APC Cy7, and anti-TRAIL-PE Abs. The levels of surface-bound TRAIL on gated viable memory B cells are shown with their representative histograms $(n=15)$. Similar results were obtained for MFI. (D) The correlation between the frequency of memory B cells and the surface expression levels of TRAIL on these cells is shown ( $n=45$; Spearman test). (E-G) Memory B cells from ST and HIV- subjects were pretreated with TRAILi and then cocultured for 7 days with autologous CD19- PBMCs $(n=5)$. ( $(\mathbf{F}$ and $\mathbf{F})$ The levels of apoptosis as measured by annexin $V$ staining $(\mathbf{E})$ and the surface expression levels of TRAIL $(\mathbf{F})$ in the presence or absence of TRAILi were assessed on memory B cells on day 7. (G) Correlation between the frequency of apoptotic memory B cells and the surface expression levels of TRAIL on these cells is also shown ( $n=10$; Spearman test).

these results demonstrated a central role for Foxo3a and most likely implicated its proapoptotic targets Bim and TRAIL in regulating memory B cell survival during HIV infection.

Foxo3a-mediated memory B cell survival in ST subjects is TRAIL dependent. We then measured the expression levels of Foxo3a target molecules endowed with proapoptotic properties in ex vivo-purified memory B cells from ST, EC, and HIV- subjects $(n=5)$. We found that memory B cells from ST subjects showed significantly higher expression levels of TRAIL $(100 \%)$ when compared with EC $(21.7 \% \pm 21 \%$; $P<0.01)$ and HIV- subjects $(19.5 \% \pm 22.7 \% ; P<0.01)$ (Figure 3, $A$ and B). Interestingly, Bim expression levels were also increased in ST subjects $(97.9 \% \pm 3.1 \%)$ when compared with EC subjects $(45 \% \pm 8.8 \%$; $P<0.01$ ) (Figure 3, A and B). However, levels of proapoptotic FasL, also regulated by Foxo3a (18), were comparable in all 3 groups
(Supplemental Figure 4). In addition, ex vivo memory B cells from ST subjects showed higher levels of expression of surface TRAIL when compared with EC and HIV- subjects using flow cytometry $(P<0.005 ; n=15)$ (Figure 3C). More importantly, surface expression levels of TRAIL on memory B cells were negatively correlated with the frequency of these cells for all subjects tested (Spearman $r=-0.4792 ; P<0.0005 ; n=45$ ) (Figure 3D). These data suggest an in vivo role of TRAIL in reducing the frequency of memory B cells in ST subjects (expressing high levels of TRAIL) when compared with those of other subjects (displaying low levels of TRAIL). The increased expression of TRAIL within ST subjects when compared with EC and HIV- subjects was specific to memory B cells, as naive $\mathrm{B}$ cells, total $\mathrm{CD}^{+} \mathrm{T}$ cells, monocytes, and plasmacytoid DCs from ST subjects displayed similar surface expression of TRAIL com- 
A

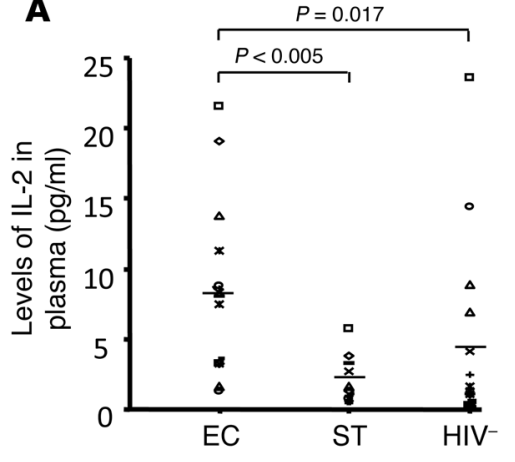

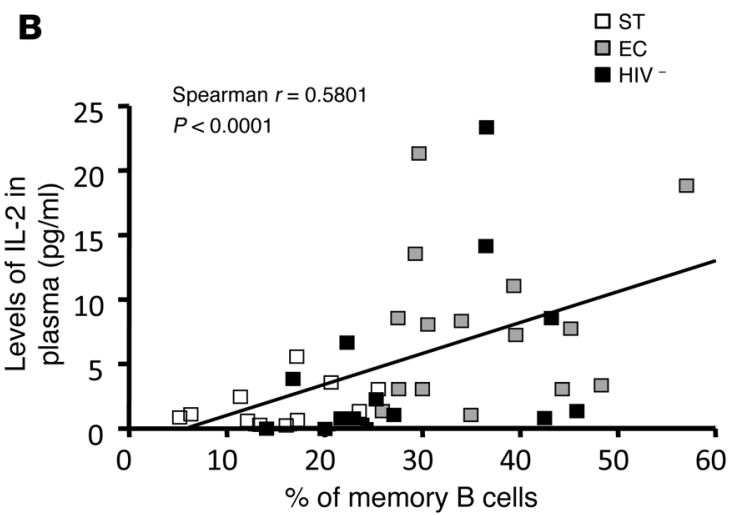

D
C

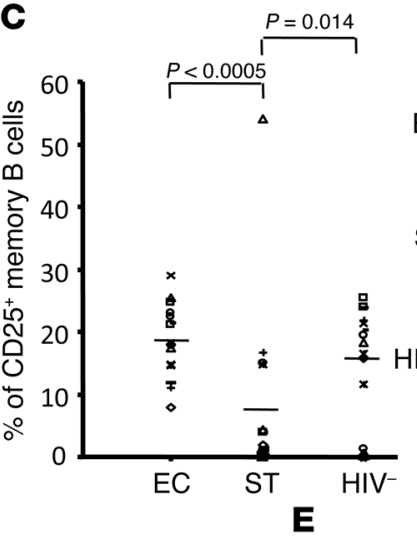

EC

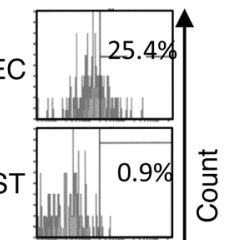

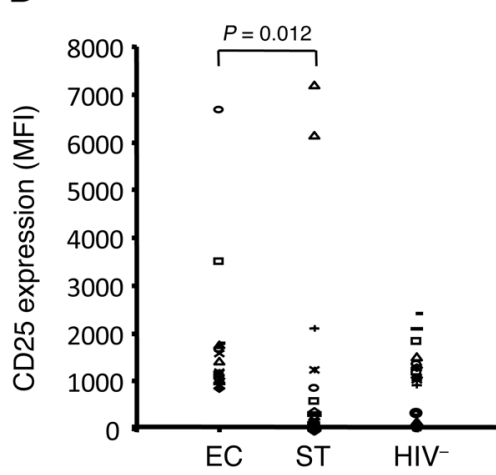

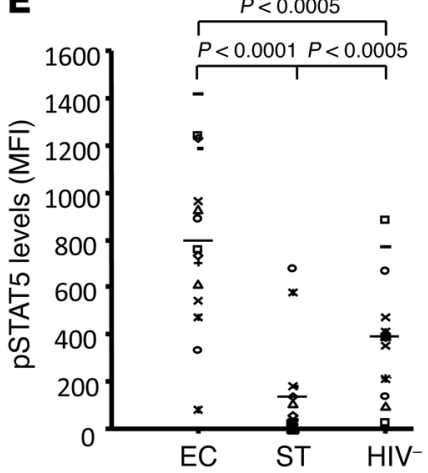

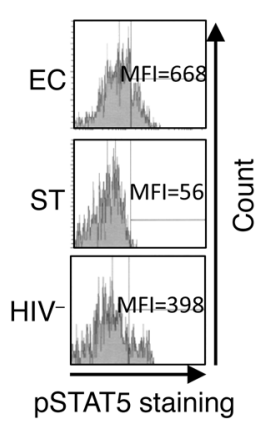

Figure 4

Reduced IL-2 signaling resulted in premature memory $B$ cell death in $\mathrm{HIV}^{+}$subjects. (A) IL-2 was measured in plasma samples from EC, ST, and HIVsubjects using ELISA $(n \geq 11$ per group). (B) Correlation between the frequency of ex vivo memory $B$ cell subsets within total $B$ cells and plasma IL-2 levels in all subjects was also determined ( $n=41$; Spearman test). (C and D) Ex vivo PBMCs from ST, EC, and HIV- subjects were stained with 7AAD, anti-CD3-PB, anti-CD19-Alexa Fluor 700, anti-CD27-APC Cy7, and anti-CD25-PE (IL-2 receptor) to monitor their surface expression levels on gated viable memory B cells $(n=15)$. Results show (C) the frequencies of positive cells and (D) the expression levels of CD25 (MFI). (E) PBMCs from $\mathrm{HIV}^{+}$ and $\mathrm{HIV}^{-}$subjects were treated for 15 minutes with $10 \mathrm{ng} / \mathrm{ml}$ IL-2, then labeled with 7AAD, anti-CD3-PB, anti-CD19Alexa Fluor 700 , and antiCD27-APC Cy7 Abs and then subjected to PhosFlow staining with anti-pSTAT5-PE $\mathrm{Ab}$. Results show the levels of pSTAT5 within viable memory $\mathrm{B}$ cells (MFI; $n=15)$. Representative histograms for Figure 3 , $\mathrm{C}$ and $\mathrm{E}$, are also shown. pared to those observed in EC and $\mathrm{HIV}^{-}$subjects (Supplemental Figure 5, A-D). Of note, we did not observe any differences in the expression levels of TRAIL receptors (DR4 and DR5) on ex vivo memory B cells between ST, EC, and $\mathrm{HIV}^{-}$subjects (Supplemental Figure 6, B and C).

To further confirm the involvement of the TRAIL pathway in reduced memory $B$ cell survival of ST subjects, we pretreated purified memory B cells from ST and HIV- subjects with TRAIL inhibitor (TRAILi) and then cocultured the cells for 7 days with autologous CD19- PBMCs. The levels of apoptosis in memory B cells were determined at day 7 using annexin V staining. The concentration of TRAILi used in our in vitro assay was first determined by its ability to inhibit TRAIL-mediated apoptosis (about 95\% inhibition) in BJAB cells (Supplemental Figure 6A and ref. 33). Our results showed that pretreatment of memory B cells with TRAILi led to a significant improvement in the survival of memory B cells from ST subjects similar to that observed in coculture of $\mathrm{HIV}^{-}$subjects $(P<0.01$; $n=5$ ) (Figure 3E). As expected, the surface expression levels of TRAIL on memory B cells in the presence or absence of TRAILi at day 7 of coculture were higher on ST subjects when compared with $\operatorname{HIV}^{-}(P<0.01 ; n=5)$ (Figure 1F). More importantly, we observed a significant positive correlation (Spearman $r=0.9543 ; P=0.0001$; $n=10)$ when we compared the expression levels of TRAIL to the levels of apoptosis of memory B cells at day 7 of coculture. Overall, these data demonstrate a key role of TRAIL in regulating peripheral memory B cell survival during HIV infection.

Levels of IL-2 in plasma positively correlate with the frequency of memory $B$ cells. Infection with HIV-1 has been previously described to alter the expression levels of several cytokines (such as IL-2, IL-15, and IL-21) involved in B cell homeostasis and maintenance (22, 34-37). In addition, $\gamma$-chain receptor cytokines such as IL-2 have been shown to inhibit Foxo3a transcriptional activity and expression of its proapoptotic target molecules (17). Since HIV infection can modulate the establishment of IL-2-producing, HIV-specific memory $\mathrm{CD}^{+} \mathrm{T}$ cells in viremic subjects $(38,39)$ as well as in aviremic treated subjects (40), we investigated whether IL-2 expression was modulated in our patient cohorts. We first measured the levels of secreted IL-2 in plasma samples collected from ST, 
A

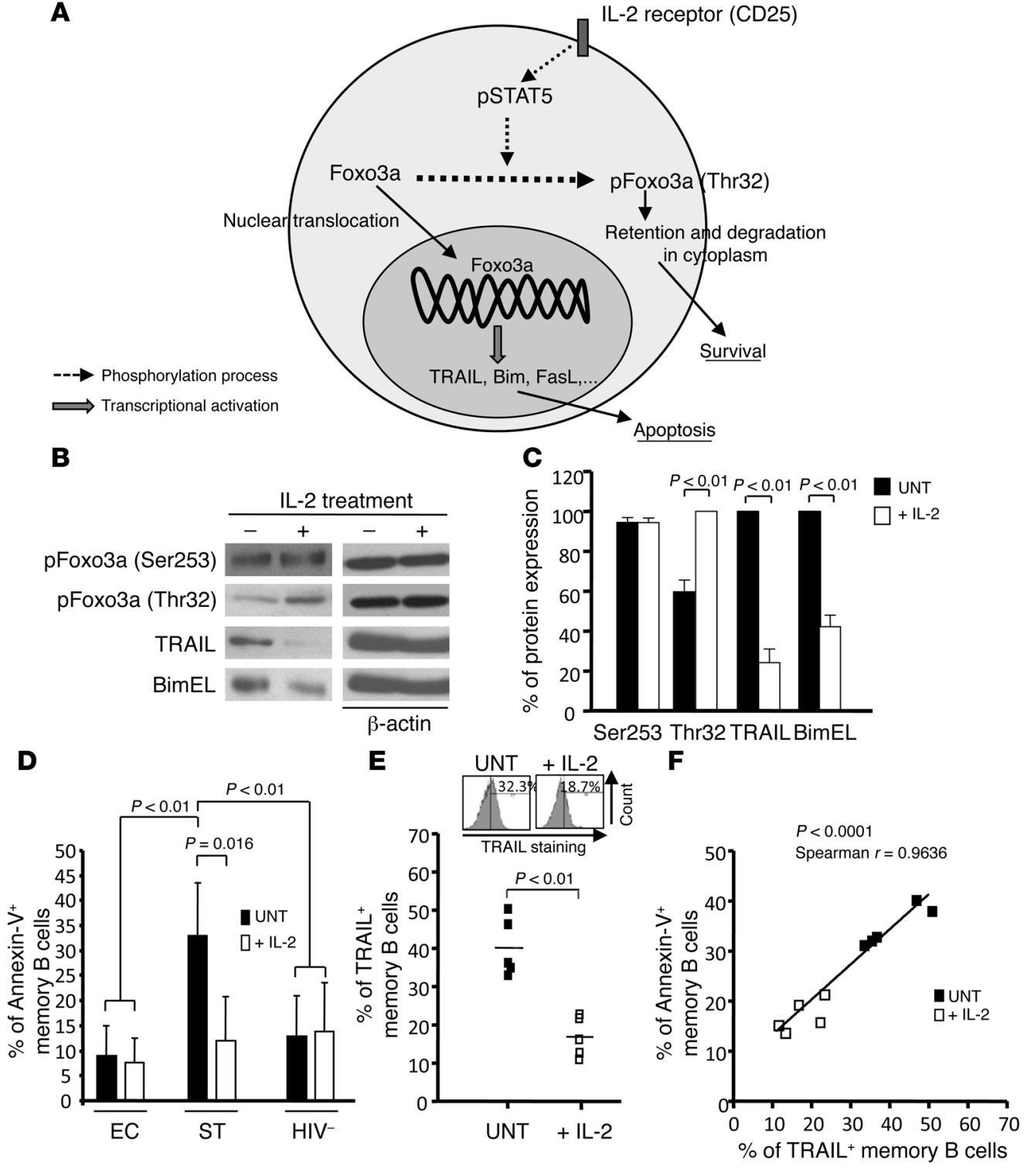

\section{Figure 5}

IL-2 treatment of memory B cells from ST subjects led to reduced levels of apoptosis by decreasing TRAIL expression. (A) Schematic showing the modulation of Foxo3a signaling pathway by IL-2 triggering. (B) Western blot analysis of memory B cells purified from ST subjects untreated or treated with $10 \mathrm{ng} / \mathrm{ml}$ of IL-2 for 15 minutes ( $p F o x o 3 a$ ) or 24 hours (BimEL and TRAIL) $(n=5)$. The expression levels of actin were measured on the same blots to control for loading. (C) Densitometric quantification of specific bands was performed using ImageQuant software (mean $\pm \mathrm{SD}$ ). (D and $\mathbf{E}$ ) Purified memory B cells from ST, EC, and HIV- subjects were cocultured for 7 days with autologous CD19- PBMCs in the presence or absence of $10 \mathrm{ng} / \mathrm{ml} \mathrm{IL-2}(n=5)$. UNT, untreated. (D) Percentage of apoptosis \pm SD on cocultured memory B cells (after 7 days) for all groups of subjects was determined by annexin V-APC. (E) Surface expression levels of TRAIL on memory B cells from ST subjects in the presence or absence of IL-2 at day 7 of coculture. Histograms shown are representative of 5 different ST subjects. (F) Correlation between surface expression of TRAIL and the percentage of apoptosis in memory B cells from ST subjects at day 7 of coculture in the presence or absence of IL-2 ( $n=10$; Spearman test).
EC, and HIV ${ }^{-}$subjects using ELISA ( $n \geq 11$ per group of subjects). We found significantly higher levels of IL-2 in the plasma of EC $(8.2 \% \pm 6.1 \%)$ when compared with ST $(2.2 \% \pm 1.7 \% ; P<0.005)$ and $\mathrm{HIV}^{-}$subjects $(4.5 \% \pm 6.6 \% ; P=0.017)(n \geq 11)$ (Figure $\left.4 \mathrm{~A}\right)$. Of note, we also observed reduced levels of IL-2 in ST subjects when compared with $\mathrm{HIV}^{-}$subjects. In addition, a significantly positive correlation (Spearman $r=0.5801 ; P<0.0001 ; n=41$ ) was observed when comparing IL-2 levels and the frequency of memory B cells in all subjects (Figure 4B), highlighting the potential involvement of IL-2 signaling in the in vivo maintenance of memory B cells.

Memory B cells from ST subjects display decreased capacity to respond to IL-2 triggering. To further examine the contribution of IL-2 signaling on memory B cell survival during HIV infection, we assessed the surface expression levels of the IL-2 receptor (CD25) in ex vivo memory B cells from all patient groups. The expression levels of CD25 on memory B cells from ST subjects were significantly lower than those from EC and HIV ${ }^{-}$subjects $(P<0.0005$ and $P=0.014$, respectively; $n=15$ ) (Figure $4 \mathrm{C}$ ). We also found decreased levels of
CD25 MFI on memory B cells from ST subjects when compared with EC subjects $(P=0.012 ; n=15)$ (Figure 4D). The reduced expression of CD25 on memory B cells from ST subjects suggested that the response of these cells to IL-2 could be affected when compared with EC and HIV- subjects. To test this hypothesis, we measured the levels of phosphorylated STAT5 (pSTAT5) in IL-2stimulated memory B cells by PhosFlow. Memory B cells from ST subjects expressed significantly lower levels of PSTAT5 in response to IL-2 when compared with those from EC and $\mathrm{HIV}^{-}(P<0.0001$ and $P<0.005$, respectively; $n=15$ ) (Figure $4 \mathrm{E}$ ). Of note, the levels of pSTAT5 were found to be significantly higher in EC when compared with $\mathrm{HIV}^{-}$subjects $(P<0.005 ; n=15)$ (Figure 4E). It is worth noting that memory B cells from ST subjects still responded to exogenous IL-2, albeit at lower levels (fold increase [FI] $1.32 \pm 0.22$ ) compared with EC and HIV ${ }^{-}$subjects (FI $1.83 \pm 0.26$ and $1.89 \pm 0.3$, respectively) (Supplemental Figure 7). These results showed that IL-2 signaling was reduced in memory B cells from ST subjects and that this could be associated with their altered survival. 
A

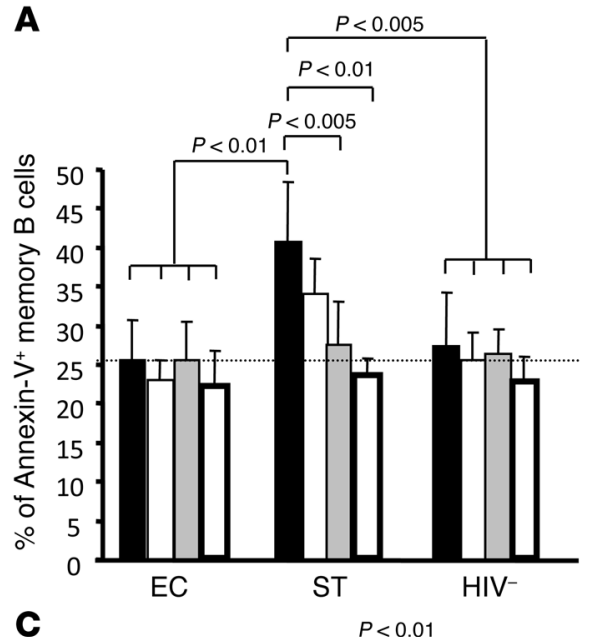

C

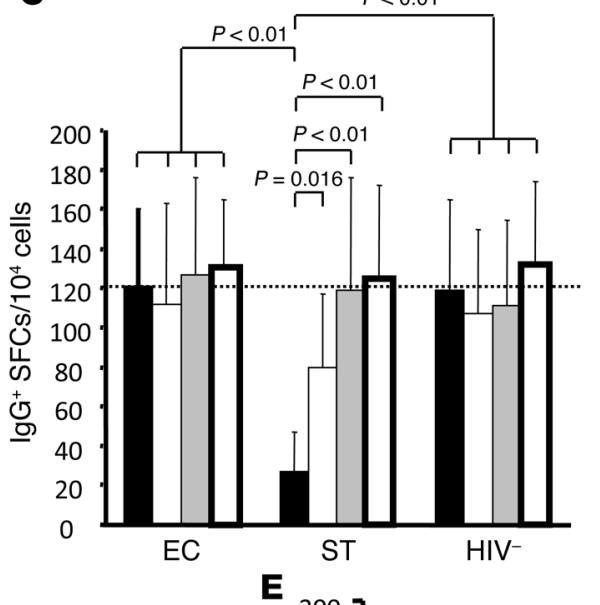

B

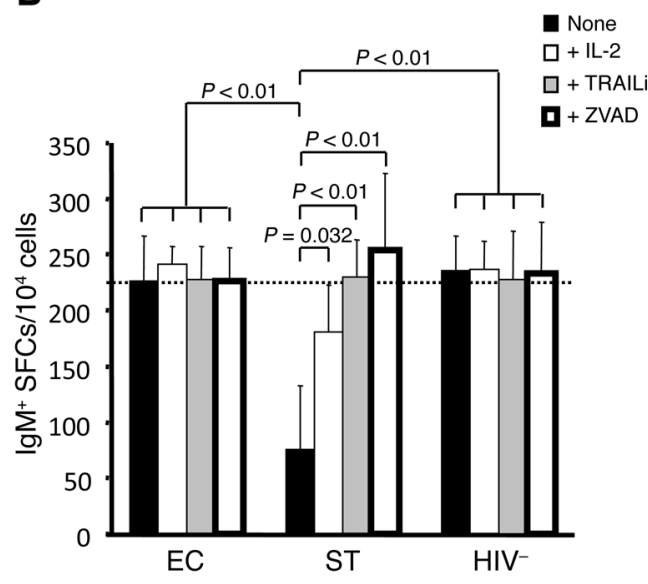

D

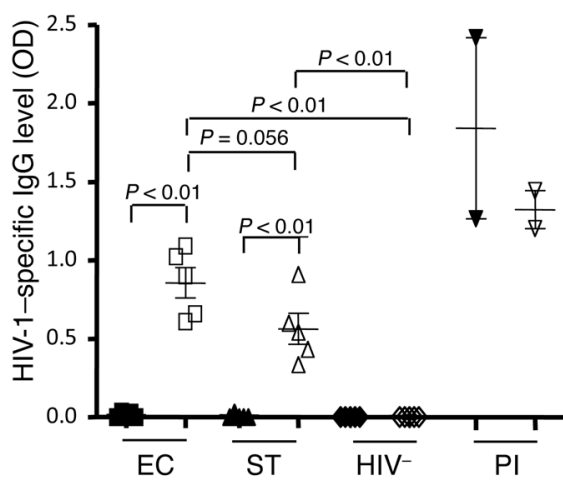

Figure 6

The improvement of ASC survival in ST subjects was achieved by enhancing memory B cell survival. Purified memory B cells from ST, EC, and $\mathrm{HIV}^{-}$subjects were polyclonally activated and cocultured for 3 days in the presence or absence of exogenous IL-2, TRAILi, or Z-VAD. (A) The percentage of apoptosis \pm SD was determined on day 3 by annexin $\mathrm{V}-$ APC staining $(n=5)$. (B and C) Memory $\mathrm{B}$ cells from $\mathrm{HIV}^{+}$and $\mathrm{HIV}^{-}$subjects were activated for 3 days in ELISPOT plates $(n=5)$. The plates were then treated with anti-IgG and anti-IgM Abs, followed by the addition of secondary alkaline phosphataseconjugated $\mathrm{Ab}$ to quantify the numbers of (B) IgM- and (C) IgG-secreting cells. Results represent the number of SFCs detected for each condition per $10^{4}$ purified memory $B$ cells (mean $\pm S D$ ). Unstimulated memory B cells were used to calculate the nonspecific background. (D) Levels of HIV-specific IgG Abs produced by PBMCs from EC, ST, and $\mathrm{HIV}^{-}$subjects activated with PWM for 3 days using ELISA assays. Results showed OD measured at $405 \mathrm{nM}$. (E) Correlations between the frequency of apoptotic memory $B$ cells and the number of $\operatorname{lgG}^{+}$ASCs after 3 days of activation are depicted for all subjects ( $n=15$; Spearman test).

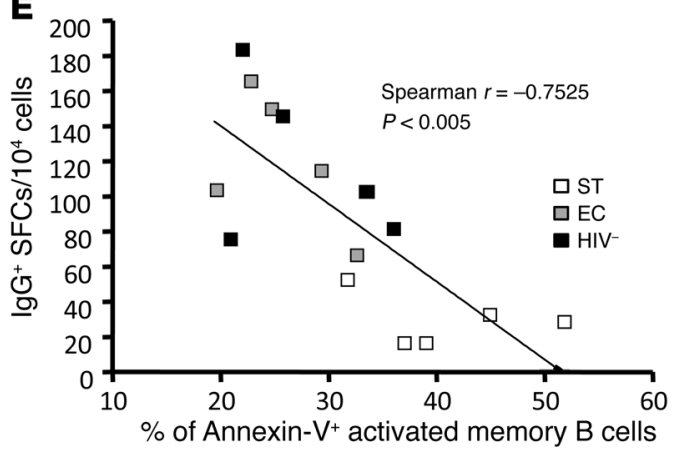

Exogenous IL-2 enhanced memory B cell survival from ST subjects by regulating Foxo3a and TRAIL pathways. We investigated whether the addition of exogenous IL-2 would enhance the survival of memory B cells from ST subjects in the 7-day coculture assay in a Foxo3a/TRAIL-dependent manner. We first determined whether IL-2 induced phosphorylated Foxo3a (pFoxo3a) in memory B cells. This has been shown to block Foxo3a transcriptional activity by excluding it from the nucleus (refs. 17, 21, and 22 and Figure 5A). Treatment of memory B cells from ST subjects with the $\gamma$-chain receptor cytokine IL-2 for 30 minutes led to a significant increase in pFoxo3a at its Thr32 residue $(P<0.01$ compared with untreated cells; $n=5$ ) (Figure 5, B and C). We did not observe differences in pFoxo3a at Ser253 in response to IL-2, as we previously reported in human memory CD4 ${ }^{+} \mathrm{T}$ cells (17). Accordingly, IL-2-treated memory B cells from ST subjects also displayed a significant decrease in TRAIL and BimEL expression levels after 24 hours $(P<0.01$ compared with untreated cells; $n=5$ ) (Figure 5, B and C).

We then determined whether exogenous IL-2 could improve memory B cell survival from ST subjects. Purified memory B cells from ST, EC and $\mathrm{HIV}^{-}$subjects were untreated or pretreated with exogenous IL-2 and then cocultured with autologous CD19PBMCs for 7 days. The levels of apoptosis, as measured by annexin $\mathrm{V}$ staining, and the surface expression levels of TRAIL were determined in memory B cells at day 7 of coculture. Treatment with IL-2 significantly enhanced survival of memory B cells from ST subjects similar to levels observed in EC and $\mathrm{HIV}^{-}$subjects $(31 \% \pm 6.4 \%$ and $11.9 \% \pm 8.9 \%$ of apoptosis for untreated and IL-2-treated cells, respectively; $P=0.016 ; n=5$ ) (Figure $5 \mathrm{D}$ ). However, no differences in B cell survival were observed when comparing IL-2-treated and 
A

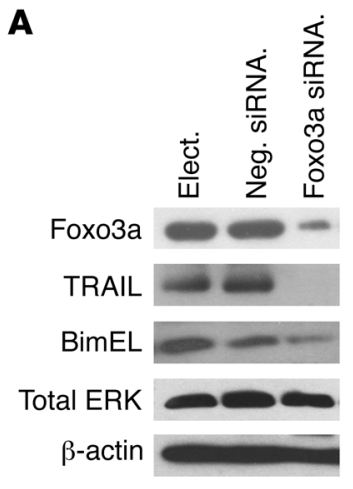

C

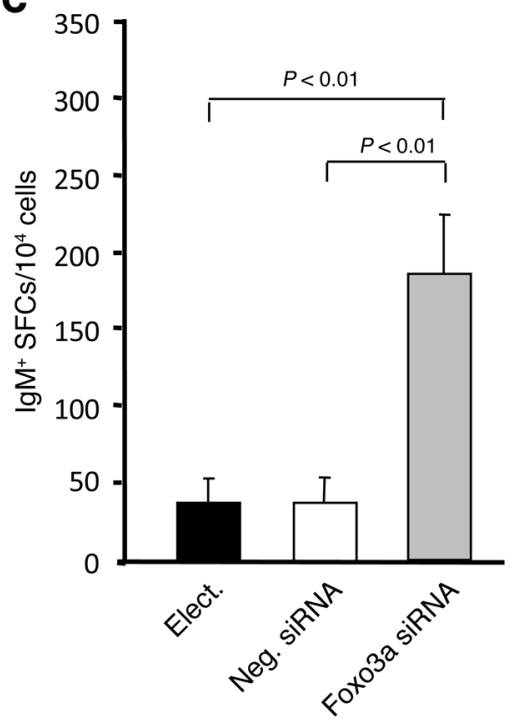

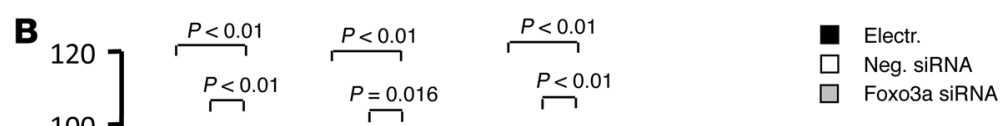

D
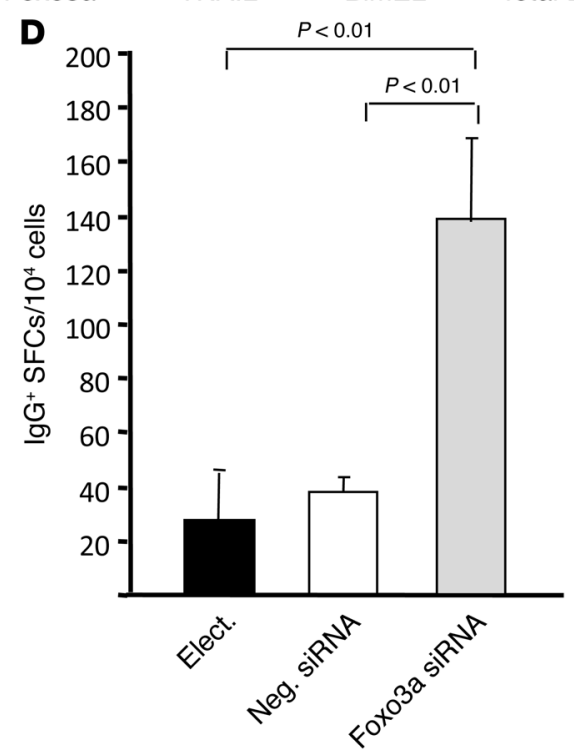

Figure 7

Silencing Foxо3a increased the number of ASCs in ST subjects. Memory B cells from EC and ST subjects were transfected with Foxo3a siRNA or negative siRNA for 2 days and then polyclonally activated for 3 days. (A) Western blot analysis of Foxo3a and its proapoptotic targets (TRAIL and Bim) was performed on memory B cells from ST subjects after 2 days of transfection and showed reduced Foxo3a, TRAIL, and Bim expression levels. (B) Densitometric values are representative of 5 independent experiments (mean \pm SD). (C and D) ELISPOT assay was performed following activation to measure (C) IgM- and (D) IgG-secreting cells as described above. Results are expressed as the mean ASC number \pm SD $(n=5)$.

untreated memory B cells in EC and $\mathrm{HIV}^{-}$subjects (Figure 5D). More importantly, IL-2 treatment of memory B cells from ST subjects led to a significant decrease in TRAIL expression levels at day 7 of coculture $(P<0.01 ; n=5)$ (Figure 5E). Accordingly, we found a highly significant positive correlation between the levels of TRAIL and the percentage of apoptosis in memory B cells from ST subjects in the presence or absence of IL-2 (Spearman $r=0.9636$; $P<0.0001 ; n=10$ ) (Figure 5F).

Overall, these findings highlight the role of decreased IL-2 signaling on the survival of memory B cells during chronic HIV infection and its downstream effects on Foxo3a transcriptional activity, such as expression of the proapoptotic molecule TRAIL. We further showed that the ex vivo defects in IL-2 signaling in these cells were still reversible despite chronic HIV-1 infection.

Increase in the number of Ab-secreting cells from ST subjects through disruption of the Foxo3a/TRAIL axis. We next determined whether there was impairment in the numbers and function of IgM and IgG Ab-secreting cells (ASCs) from HIV-infected and healthy subjects upon polyclonal activation. Briefly, purified memory B cells from ST, EC, and HIV- subjects were polyclonally activated and cocultured for 3 days with autologous CD19- PBMCs. Using annexin $\mathrm{V}$ staining, activated memory B cells from ST subjects at day 3 of coculture exhibited significantly higher levels of apoptosis $(40.7 \% \pm 7.7 \%)$ when compared with EC $(25.6 \% \pm 5.2 \%$; $P=0.016 ; n=5)$ and $\mathrm{HIV}^{-}$subjects $(27.4 \% \pm 6.8 \% ; P<0.005)$ (Figure 6A). Accordingly, by using ELISPOT assays, cocultures from ST subjects showed lower numbers of $\operatorname{IgM}^{+}$and $\mathrm{IgG}^{+}$ASCs (expressed as number of spot-forming cells [SFCs] per $10^{4}$ cultured cells) compared with those from EC and HIV ${ }^{-}$subjects $(P<0.01$; $n=5$ ) (Figure 6, B and C). Similar results were also observed when we compared the levels of HIV-1 Gag- and ENV-specific Abs in PBMCs following activation with pokeweed mitogen (PWM). As reported above for total immunoglobulin, ST subjects produced lower levels of HIV-specific Abs when compared with EC subjects $(P=0.056$; fold decrease, $1.52 \pm 0.99 ; n=5)$ (Figure 6D), indicating that the production of both HIV-and non-HIV-specific immunoglobulins was lower in ST subjects when compared with EC and HIV- subjects. 
We further determined whether the improvement of activated memory B cell survival would lead to a concomitant increase in the levels of $\mathrm{Ab}$ production. Treatment of memory B cells from ST subjects with Z-VAD (Pan caspase inhibitor) or with TRAILi significantly enhanced their survival (FI $1.7 \pm 0.2[P<0.01]$ and $1.5 \pm 0.5[P=0.032]$, respectively) (Figure 6A). Treatment with exogenous IL-2 also led to a slight increase in memory B cell survival in ST subjects (FI 1.2 \pm 0.1 ) (Figure 6A). Subsequently, this led to a significant increase in the numbers of ASCs $\left(\operatorname{IgM}^{+}\right.$ASCs: Z-VAD, FI $4.9 \pm 3$, $P<0.01$; TRAILi, FI $4.5 \pm 2.6, P<0.01$; exogenous IL-2, FI $3.6 \pm 2.1$, $P=0.032$; IgG ${ }^{+}$ASCs: Z-VAD, FI $4.5 \pm 2, P<0.01$; TRAILi, FI $4.4 \pm 2.6$, $P<0.01$; exogenous IL-2, FI $3.1 \pm 2.1, P=0.016$ ) (Figure $6, \mathrm{~B}$ and C). Treatment of memory B cells from EC and HIV- subjects with these exogenous molecules did not improve the survival rates of these cells or the number of ASCs generated after 3 days of activation (Figure 6, A-C). Of note, we found a significant inverse correlation when we compared the frequency of annexin $\mathrm{V}^{+}$memory $\mathrm{B}$ cells with the number of $\mathrm{IgG}^{+}$ASCs after 3 days of activation within all studied subjects (Spearman $r=-0.7525 ; P<0.005 ; n=15$ ) (Figure 6E). Moreover, we found similar levels of proliferation in viable activated memory B cells from ST, EC, and $\mathrm{HIV}^{-}$subjects, as measured by CFSE dilution (Supplemental Figure 8). This is in accordance with results from previous studies $(5,41,42)$, confirming that the decreased number of ASCs in ST subjects was not due to lower levels of proliferation but was the result of reduced survival of activated memory B cells.

To further establish a direct role of Foxo3a and TRAIL in regulating the numbers of ASCs, purified memory B cells from ST subjects were transfected with Foxo3a siRNAs or control siRNAs for 2 days prior to activation. We obtained high levels of Foxo3a silencing in memory B cells after 2 days of siRNA transfection (Figure 7, A and B). Cells were subsequently activated for 3 days by polyclonal activation, as described for Figure 6, B and C. The results shown in Figure 7, C and D, indicated that decreased expression of Foxo3a led to a significant increase of ASC numbers in ST subjects (FI $7 \pm 5.7$ and $3.7 \pm 1$ for $\mathrm{IgM}^{+}$and $\mathrm{IgG}^{+} \mathrm{ASCs}$ from ST subjects, respectively; $P<0.01 ; n=5)$. Our data thus confirmed that Foxo3a-induced apoptosis in ASCs from ST subjects was TRAIL dependent, as Foxo3a silencing significantly reduced TRAIL expression (Figure 7, A and B).

Altogether these results confirm that activated memory B cells from ST subjects show a decrease in ASC persistence. Moreover, we showed that the reduced production of Abs observed in ST subjects was associated with higher levels of caspase-mediated apoptosis within memory B cells and was restored by silencing Foxo3a, inhibiting TRAIL, or adding exogenous IL-2 to levels similar to those produced by EC and $\mathrm{HIV}^{-}$subjects.

\section{Discussion}

Herein we show, for what we believe is the first time in human subjects, the critical implication of Foxo3a and its transcriptional target TRAIL in memory $\mathrm{B}$ cell survival and $\mathrm{Ab}$ production during HIV-1 infection. We show that higher Foxo3a transcriptional activity was observed in ex vivo peripheral memory B cells from ST subjects when compared with EC and HIV-1- ${ }^{-}$subjects, as these cells express heightened levels of TRAIL and Bim (Figure 3, A-C). Silencing Foxo3a by siRNA or interfering with TRAIL signaling in memory B cells from ST subjects significantly enhanced their in vitro survival to levels comparable to those observed in EC and $\mathrm{HIV}^{-}$subjects (Figures 2, 3, 6, and 7).
Foxo3a transcriptional activity is regulated at multiple levels including phosphorylation, acetylation, and ubiquitination (18). It would be difficult to determine which of these mechanisms is at play in the context of chronic HIV-1 infection. Nonetheless, despite similar expression levels in total Foxo3a and pFoxo3a at Ser253 in ex vivo memory B cells from ST, EC, and $\mathrm{HIV}^{-}$subjects, we found a significant decrease in the expression levels of pFoxo3a at Thr32 in ST subjects $(20.5 \% \pm 22.7 \%)$ when compared with EC subjects $(81 \% \pm 22.7 \%$; $P=0.016 ; n=5$ ) (data not shown). We also observed lower levels of pFoxo3a at Thr32 in ST subjects when compared with HIV- subjects, although this difference was not significant $(63.9 \% \pm 48.1 \%)$ (data not shown). These results suggest that the regulation of Foxo3a transcriptional activity might be mediated by more than 1 mechanism and might involve multiple integrated signals.

Of note, TRAIL has been described as a downstream target of type I interferons (43) whose expression levels are known to be upregulated during HIV infection (44). As shown here, TRAIL is also a transcriptional target of Foxo3a; our preliminary data thus established a link between Foxo3a dephosphorylation and interferon within memory B cells (Supplemental Figure 9). Although the present study clearly implicated TRAIL in the reduced survival observed in memory B cells from ST subjects, the correlation between the surface expression levels of TRAIL and the frequencies of memory B cells for all subjects tested (Figure 3D; where the $P$ value was driven mainly by high and low levels of TRAIL) suggested that other factors such as Bim, FasL, NOXA, or PUMA known transcriptional targets of Foxo3a (18-20) - might be involved. This observation is further supported by the fact that we did not observe any correlation between these 2 parameters in the ST group (data not shown). Similarly, it is possible that other forkhead family members such as Foxo1a, whose activity is also regulated by IL-2 (45), might be involved.

Several factors known to be associated with HIV pathogenesis are also involved in regulating the phosphorylation of Foxo3a and the expression of its transcriptional targets. In this context, hyperimmune activation (a hallmark of HIV infection) is associated with increased levels of interferons (43), inflammatory cytokines (46-48), TGF- $\beta 1$ (49), and products of bacterial translocation (50). Interestingly, our preliminary results on purified memory B cells showed that in vitro stimulation with type I and II interferons as well as TNF- $\alpha$ and TGF- $\beta 1$ induced the expression of Foxo3a proapoptotic target genes (Supplemental Figure 9). Conversely, molecules such as IL-21 and IL-15, whose expression levels are reduced during HIV infection $(35,36)$, phosphorylated Foxo3a and downregulated the transcription of its proapoptotic target BimEL (Supplemental Figure 10).

Interestingly, an important role for the PD-1 pathway, known to inhibit PI3K/AKT phosphorylation (51), has been recently reported to be involved in the depletion of memory B cells and to impair humoral responses in SIV-infected macaques (52). This evidence suggests that PD-1 signaling might modulate Foxo3a transcriptional activity in memory B cells from HIV-infected subjects. Foxo3a could thus integrate distinct signals that are aberrantly expressed in HIV infection; this would subsequently affect its proapoptotic transcriptional activity via the induction of TRAIL and/or other molecules, leading to the reduced survival levels observed in memory B cells in infected subjects.

In this study we demonstrated that the reduced levels of peripheral memory B cells observed in ST subjects were associated with decreased IL-2 signaling (Figures 4-6). Interestingly, IL-2 was pre- 
viously shown to be involved in the activation of memory B cells (53) and in the enhanced production of anti-HIV Abs $(54,55)$. IL-2 was also shown to be involved in increased $\mathrm{Ab}$ responses to tetanus vaccination in IL-2-treated ST subjects (56), confirming the involvement of this cytokine in the persistence and development of B cell responses. However, despite the significant in vitro improvement of memory B cell and ASC survival in ST subjects upon exogenous IL-2 stimulation, the restoration was not complete (around 65\%; Figure 5D). These results strongly suggest that other upstream signals besides IL-2 may contribute to the alteration of Foxo3a activity and to the altered maintenance of memory B cells in chronic HIV-infected subjects.

The extent of restoration of the humoral immune response following HAART has been shown to be dependent on the early initiation of treatment. Recently, Moir et al. showed that early initiation of antiretroviral therapies within the first year restored the frequency of memory B cells to levels observed in healthy individuals (57). Similarly, Chong et al. confirmed these results, although the data also suggested that with time, despite early treatment with antiretroviral therapy, there was a decline in the humoral immune response (10). Our own subjects, who had been treated early and for at least 5 years, showed regression of memory B cells that was not fully restored by HAART. Altogether, these results seem to suggest that the early initiation of HAART temporally restores memory B cell numbers but may be unable to halt the slow progressive decline over time. This is in accordance with the fact that progressive and persistent structural and anatomic damages occur in lymph nodes within the first 2 weeks of HIV infection (site of memory B cell generation) and are not fully restored by $\operatorname{HAART}(8,58)$.

In addition, our results also highlight the fact that memory $B$ cells from EC subjects were significantly different than those from ST subjects and, more interestingly, displayed unique characteristics that were not always found in $\mathrm{HIV}^{-}$subjects. We found that the ex vivo frequencies of memory B cells (Figure 1C), the levels of IL-2 in their plasma (Figure 4A), and the responsiveness to IL-2 as measured by pSTAT5 levels (Figure 4E) were significantly higher in EC subjects when compared not only with ST subjects but also with $\mathrm{HIV}^{-}$, suggesting an increased survival capability of memory B cells within EC subjects. Of note, the higher frequencies of memory B cells observed within EC subjects could not be explained by previously reported polymorphisms such as CCR5 mutations associated with HIV protection (Supplemental Table 1 and ref. 15).

Overall, these data confirmed that, despite HAART, there is still a requirement to maintain the integrity and survival of peripheral $\mathrm{CD} 27^{+}$memory B cells to prevent HIV disease progression and thereby enhance the success of possible antiviral vaccines. The manipulation or induction of Foxo3a activity by anticancer drugs such as imatinib or the use of recombinant TRAIL has proven efficient in treating resistant cancers and autoimmune diseases (59-61). Conversely, interfering with Foxo3a and TRAIL signaling, alone or in combination with HAART, could rescue both $\mathrm{T}$ and $\mathrm{B}$ cell functions and could also restore global $\mathrm{Ab}$ responses including those elicited against HIV, thus improving the success of therapeutic vaccines against this and other infections.

\section{Methods}

Products. RPMI-1640 media, FBS, and $\beta$-mercaptoethanol were purchased from Sigma-Aldrich. All Abs used for flow cytometry were purchased from BD Biosciences, except for anti-DR4 and anti-DR5 Abs, which were obtained from Abcam. All primary Abs used for Western blots (anti-phospho forms of Foxo3a, anti-Bim, and anti-ERK) were purchased from Cell Signaling. Anti-actin Abs were purchased from Sigma-Aldrich, anti-Foxo3a from Abcam, and anti-TRAIL from ProSci Inc. Secondary HRP-conjugated goat anti-mouse and anti-rabbit IgG Abs were obtained from Bio-Rad Laboratories. IL-2, IL-6, and IL-10 cytokines were purchased from R\&D Systems. Anti-CD40 Ab (clone G28.5; ATCC hybridoma) was provided by Walid Mourad (Centre Hospitalier de l'Université de Montréal).

Purification of $\mathrm{CD} 27^{+}$memory $\mathrm{B}$ cells. Memory B cells were purified using magnetic bead separation (Miltenyi Biotec). We performed an initial negative selection step using B Cell Isolation Kit 2, which allowed us to retain total B cells, followed by a positive selection step using CD27 microbeads (Miltenyi Biotec), which allowed us to purify the $\mathrm{CD} 27^{+}$memory B cell subset. To achieve the highest purity of memory B cells from our subject cohorts, we performed 3 rounds of elution for both the negative and positive selection steps. In addition, purified memory B cells from all subject groups were analyzed for purity, and levels of apoptosis using CD3-CD19+ and annexin $\mathrm{V}$ phenotype. The purity of memory $\mathrm{B}$ cells was determined at around $93.6 \%$ using flow cytometry. Equal numbers of memory B cells from the 3 subject cohorts were used in the coculture system. This was done by cell counting with trypan blue exclusion.

STAT5 phosphorylation assay. PBMCs from ST, EC, and $\mathrm{HIV}^{-}$subjects were stimulated with $10 \mathrm{ng} / \mathrm{ml}$ of IL-2 for 15 minutes. STAT5a phosphorylation was measured with BD Biosciences Phosflow using anti-pSTAT5a specific $\mathrm{Ab}$ as previously described (17).

Western blots. Highly purified sorted CD3-CD $19^{+} \mathrm{CD} 27^{+}$memory B cells from $\mathrm{HIV}^{+}$and $\mathrm{HIV}^{-}$subjects were subjected to SDS-PAGE and Western blot analysis as previously described $(15,17)$.

Memory B cell coculture assay. Purified memory B cells $\left(10^{5}\right)$ were cocultured for 7 days with $9 \times 10^{5}$ autologous CD19-depleted PBMCs (purity >99.8\%) in the presence of $10 \mu \mathrm{M}$ azidothymidine to inhibit the production of HIV viral particles. P24 ELISA performed on the supernatant confirmed the lack of viral replication. Absolute numbers of viable memory B cells and the frequency of apoptosis (annexin $\mathrm{V}^{+}$cells) were measured at day 7 of coculture.

Transfection of siRNA in the 7-day coculture assay. Purified memory B cells $\left(6 \times 10^{6}\right)$ were electroporated using Nucleofector II technology according to the Amaxa Biosystems manufacturer's protocol. Specific Foxo3a siRNA and siRNA negative control were obtained from Invitrogen (Foxo3a Validated Stealth DuoPak). siRNA ( $3 \mu \mathrm{g})$ was transfected for each condition. Following transfection, cells were cultured in RPMI media without antibiotics for 6 hours. Cells were then washed at $224 \mathrm{~g}$ for 10 minutes at room temperature (RT) to remove dead necrotic cells. Transfected cells were then washed, counted, and cocultured with their autologous CD19- PBMCs for 7 days. Two days onto the coculture assay, cells were purified by negative selection using a $\mathrm{B}$ cell isolation kit and assayed for Foxo3a, BimEL, TRAIL, total ERK, and $\beta$-actin expression through Western blot analysis.

Polyclonal activation. CFSE-labeled purified memory B cells were first polyclonally activated for 2 hours with $5 \mu \mathrm{g} / \mathrm{ml}$ anti-CD $40,0.1 \mu \mathrm{g} / \mathrm{ml}$ antiIgG plus anti-IgM (Jackson ImmunoResearch; catalog no. 109-005-044), $10 \mathrm{ng} / \mathrm{ml}$ cytokines (IL-6 plus IL-10) in the presence or absence of IL-2 $(10 \mathrm{ng} / \mathrm{ml})$, TRAILis $(20 \mathrm{ng} / \mathrm{ml})$, or Z-VAD $(25 \mu \mathrm{M})$. Activated memory $B$ cells were washed and cocultured for 3 days with autologous CD19PBMCs to generate ASCs in the presence of AZT. P24 ELISA performed on the supernatant confirmed the lack of viral replication. Apoptosis was performed at day 3 on gated activated memory B cells using annexin V. DMSO $(0.01 \%)$ was included in these experiments as negative control and gave similar results to untreated conditions.

In some experiments (Figure 7, A-D), cells were transfected as described above and then cocultured with autologous CD19- depleted PBMCs for 2 days. This ensured efficient Foxo3a silencing within memory B cells prior to their activation. Memory B cells were then purified at day 2 using a B 
cell isolation kit and assessed for Foxo3a, BimEL, TRAIL, total ERK, and $\beta$-actin expression by Western blot analysis. Transfected purified memory $\mathrm{B}$ cells were also polyclonally activated (as described above) for an extra 3 days in ELISPOT plates to measure ASC generation.

ELISPOT assay. The numbers of ASCs were measured on day 3 activated memory B cells by ELISPOT assay according to the manufacturer's protocol (ELISPOTplus for human IgM and IgG, 3840-2AW-Plus and 3820-2AWPlus, respectively; MABTECH). SFCs generated from activated and treated memory B cells from ST, EC, and HIV- subjects were enumerated with ImmunoSpot S5 UV Analyzer (Cellular Technology Ltd.) and normalized to their negative control (resting memory B cells from the same patient).

$H I V$-specific ELISA. PBMCs from ST, EC, $\operatorname{HIV}^{-}(n=5)$, and primary infected (PI; $n=2$ ) subjects were polyclonally activated with or without $1 \mu \mathrm{g} / \mathrm{ml}$ PWM for 3 days. Supernatants $(200 \mu \mathrm{l})$ were collected to measure HIV-1-specific IgG Ab titers using ELISA. To that aim, $1 \mu \mathrm{g}$ of HIV-1 Gag (HIV-121) and $1 \mu \mathrm{g}$ of ENV (HIV-101) (Prospect Proteins Inc.) diluted in coating Buffer (eBioscience) were incubated overnight at $4^{\circ} \mathrm{C}$ on 96 -well plates. The next day, plates were washed 10 times in PBS/0.05\% Tween20. This was followed by 30 minutes of saturation in PBS/10\% FBS. Plates were then incubated with culture supernatants for 2 hours at RT, washed 10 times, and incubated for 2 hours at RT with $100 \mu \mathrm{l} 1 \mu \mathrm{g} / \mathrm{ml}$ anti-IgG-biotin (ELISPOT kit for human IgG, 3850-2HW-Plus; Mabtech). Plates were washed 10 times and incubated 1 hour at RT with $100 \mu$ l diluted streptavidin-HRP (1:1,000 in PBS) (3850-2HW-Plus; Mabtech). Following a final wash step in PBS/0.05\% Tween-20, $100 \mu \mathrm{l}$ $1 \times$ TMB substrate solution (eBioscience) were added per well until appearance of color. Enzymatic reactions were stopped by adding $50 \mu \mathrm{l}$ $1 \mathrm{M} \mathrm{H}_{3} \mathrm{PO}_{4}$. Finally, OD at $405 \mathrm{nM}$ was measured with SpectraMax plus 384-plate reader (Molecular Devices).

Statistics. All statistical analyses were performed using the nonparametric Mann-Whitney $U$ test, assuming independent samples. This test, which uses the rank of the data rather than their raw values to calculate the statistical significance, is an alternative to the $t$ test when the assumption of normality is not satisfied or could not be tested in the case of small sample size. In this study, $P$ values of less than 0.05 were considered significant.

Study approval. All HIV-infected subjects provided written informed consent. This research was approved by the Office of Research Ethics, Royal Hospital, McGill University Health Center, and by the Comité d'Ethique de la Recherche et le Centre Hospitalier de l'Université de Montréal. Virological and immunological profiles for all $\mathrm{HIV}^{+}$subjects included in this study are summarized in Supplemental Table 1.

\section{Acknowledgments}

We are grateful to the patients participating in the Canadian cohort of HIV-infected subjects, their physicians, and attending staff members. We are thankful to M. Legault from the Réseau SidaMI/FRSQ for administrative and clinical coordinating support. We thank V. Lafontaine, N. Sawyer, L. Lejeune, and Y. Chouikh for their technical expertise. J. van Grevenynghe is a fellow of the Canadian Institutes of Health Research (CIHR). This study was supported by research funds from the NIH, the CIHR, Genome Quebec, Genome Canada, Fonds de Recherche en Santé du Quebec (FRSQ), and the Canadian Network for Vaccines and Immunotherapeutics. R.-P. Sékaly is a holder of the Canada Research Chair in Human Immunology. J.-P. Routy and C.L. Tremblay are clinician-scientists supported by FRSQ. Moreover, we would like to thank P. Ancuta, Y. Peretz, and J. Schatzle for critically reviewing the manuscript.

Received for publication May 27, 2011, and accepted in revised form August 3, 2011.

Address correspondence to: Elias K. Haddad or Rafick-Pierre Sékaly, 11350 SW Village Parkway, 3rd Floor, Port St. Lucie, Florida 34987, USA. Phone: 772.971.5099; Fax: 772.345.3675; E-mail: ehaddad@ vgtifl.org (E.K. Haddad). Phone: 772.345.4785; Fax: 772.345.3675; E-mail: sekaly@vgtifl.org (R.-P. Sékaly).
1. Moir S, Fauci AS. B cells in HIV infection and disease. Nat Rev Immunol. 2009;9(4):235-245.

2. De Milito A, et al. Mechanisms of hypergammaglobulinemia and impaired antigen-specific humoral immunity in HIV-1 infection. Blood. 2004; 103(6):2180-2186.

3. Amadori A, et al. B cell activation during HIV-1 infection. II. Cell-to-cell interactions and cytokine requirement. J Immunol. 1991;146(1):57-62.

4. Samuelsson A, Brostrom C, van Dijk N, Sonnerborg A, Chiodi F. Apoptosis of CD4+ and CD19+ cells during human immunodeficiency virus type 1 infection--correlation with clinical progression, viral load, and loss of humoral immunity. Virology. 1997;238(2):180-188.

5. Jiang W, et al. Impaired naive and memory B-cell responsiveness to TLR9 stimulation in human immunodeficiency virus infection. J Virol. 2008; 82(16):7837-7845.

6. Moir S, Fauci AS. Pathogenic mechanisms of Blymphocyte dysfunction in HIV disease. J Allergy Clin Immunol. 2008;122(1):12-19.

7. De Milito A, Morch C, Sonnerborg A, Chiodi F. Loss of memory (CD27) B lymphocytes in HIV-1 infection. Aids. 2001;15(8):957-964.

8. Levesque MC, et al. Polyclonal B cell differentiation and loss of gastrointestinal tract germinal centers in the earliest stages of HIV-1 infection. PLoS Med. 2009;6(7):e1000107.

9. Titanji K, et al. Primary HIV-1 infection sets the stage for important B lymphocyte dysfunctions. AIDS. 2005;19(17):1947-1955.

10. Chong Y, et al. Selective CD27+ (memory) B cell reduction and characteristic $B$ cell alteration in drug-naive and HAART-treated HIV type 1infected patients. AIDS Res Hum Retroviruses. 2004; 20(2):219-226.

11. Hart M, et al. Loss of discrete memory B cell subsets is associated with impaired immunization responses in HIV-1 infection and may be a risk factor for invasive pneumococcal disease. J Immunol. 2007; 178(12):8212-8220.

12. Longwe H, Gordon S, Malamba R, French N Characterising B cell numbers and memory B cells in HIV infected and uninfected Malawian adults. BMC Infect Dis. 2010;10:280.

13. Titanji K, et al. Loss of memory B cells impairs maintenance of long-term serologic memory during HIV-1 infection. Blood. 2006;108(5):1580-1587.

14. Cao Y, Qin L, Zhang L, Safrit J, Ho DD. Virologic and immunologic characterization of long-term survivors of human immunodeficiency virus type 1 infection. N Engl J Med. 1995;332(4):201-208.

15. van Grevenynghe J, et al. Transcription factor FOXO3a controls the persistence of memory CD4(+) T cells during HIV infection. Nat Med. 2008; 14(3):266-274.

16. Wang $Q$, et al. High level serum neutralizing antibody against HIV-1 in Chinese long-term non-progressors. Microbiol Immunol. 2008;52(4):209-215.

17. Riou C, et al. Convergence of TCR and cytokine signaling leads to $\mathrm{FOXO} 3 \mathrm{a}$ phosphorylation and drives the survival of CD4+ central memory $\mathrm{T}$ cells. J Exp Med. 2007;204(1):79-91.

18. Calnan DR, Brunet A. The FoxO code. Oncogene. 2008;27(16):2276-2288.

19. Dabrowska A, Kim N, Aldovini A. Tat-induced FOXO3a is a key mediator of apoptosis in HIV-1- infected human CD4+ T lymphocytes. J Immunol. 2008;181(12):8460-8477.

20. Sakoe Y, Sakoe K, Kirito K, Ozawa K, Komatsu N. FOXO3A as a key molecule for all-trans retinoic acid-induced granulocytic differentiation and apoptosis in acute promyelocytic leukemia. Blood. 2010;115(18):3787-3795

21. Bilancio A, et al. Key role of the p110delta isoform of PI3K in B-cell antigen and IL-4 receptor signaling: comparative analysis of genetic and pharmacologic interference with p110delta function in B cells. Blood. 2006;107(2):642-650.

22. van Grevenynghe J, et al. Lymph node architecture collapse and consequent modulation of FOXO3a pathway on memory T- and B-cells during HIV infection. Semin Immunol. 2008;20(3):196-203.

23. Brunet A, et al. Akt promotes cell survival by phosphorylating and inhibiting a Forkhead transcription factor. Cell. 1999;96(6):857-868.

24. Hu MC, et al. IkappaB kinase promotes tumorigenesis through inhibition of forkhead FOXO3a. Cell. 2004;117(2):225-237.

25. Herzog S, et al. SLP-65 regulates immunoglobulin light chain gene recombination through the PI(3)K-PKB-Foxo pathway. Nat Immunol. 2008; 9(6):623-631.

26. Herzog S, Reth M, Jumaa H. Regulation of B-cell proliferation and differentiation by pre-B-cell receptor signalling. Nat Rev Immunol. 2009;9(3):195-205.

27. Hinman RM, Nichols WA, Diaz TM, Gallardo TD, Castrillon DH, Satterthwaite AB. Foxo3 ${ }^{-/-}$mice demonstrate reduced numbers of pre- $B$ and recirculating B cells but normal splenic B cell sub-population distribution. Int Immunol. 2009;21(7):831-842. 
28. Scheeren FA, et al. STAT5 regulates the self-renewal capacity and differentiation of human memory B cells and controls Bcl-6 expression. Nat Immunol. 2005;6(3):303-313.

29. Vikstrom I, et al. Mcl-1 is essential for germinal center formation and B cell memory. Science. 2010; 330(6007):1095-1099.

30. Sanz I, Wei C, Lee FE, Anolik J. Phenotypic and functional heterogeneity of human memory B cells. Semin Immunol. 2008;20(1):67-82.

31. Klein U, Kuppers R, Rajewsky K. Evidence for a large compartment of IgM-expressing memory B cells in humans. Blood. 1997;89(4):1288-1298.

32. Weller S, et al. Human blood IgM "memory" B cells are circulating splenic marginal zone B cells harboring a prediversified immunoglobulin repertoire. Blood. 2004;104(12):3647-3654.

33. Sprick MR, et al. FADD/MORT1 and caspase- 8 are recruited to TRAIL receptors 1 and 2 and are essential for apoptosis mediated by TRAIL receptor 2 . Immunity. 2000;12(6):599-609.

34. Avery DT, et al. B cell-intrinsic signaling through IL-21 receptor and STAT3 is required for establishing long-lived antibody responses in humans. J Exp Med. 2010;207(1):155-171.

35. Biancotto A, et al. Abnormal activation and cytokine spectra in lymph nodes of people chronically infected with HIV-1. Blood. 2007;109(10):4272-4279.

36. Iannello A, et al. Dynamics and consequences of IL-21 production in HIV-infected individuals: a longitudinal and cross-sectional study. J Immunol. 2010; 184(1):114-126.

37. Litjens NH, Huisman M, Hijdra D, Lambrecht BM, Stittelaar KJ, Betjes MG. IL-2 producing memory $\mathrm{CD} 4+\mathrm{T}$ lymphocytes are closely associated with the generation of IgG-secreting plasma cells. J Immunol. 2008;181(5):3665-3673.

38. Harari A, Petitpierre S, Vallelian F, Pantaleo G Skewed representation of functionally distinct populations of virus-specific CD4 T cells in HIV-1-infected subjects with progressive disease: changes after antiretroviral therapy. Blood. 2004;103(3):966-972.

39 . Younes SA, et al. HIV-1 viremia prevents the establishment of interleukin 2-producing HIV-specific memory CD4+ T cells endowed with proliferative capacity. J Exp Med. 2003;198(12):1909-1922.

40. Ferre AL, et al. HIV controllers with HLA-DRB $1 * 13$ and HLA-DQB1*06 alleles have strong, polyfunctional mucosal CD4+ T-cell responses. J Virol. 2010;84(21):11020-11029.

41. Malaspina A, et al. CpG oligonucleotides enhance proliferative and effector responses of B cells in HIV-infected individuals. I Immunol. 2008 ; 181(2):1199-1206.

42. Mehta N, et al. Impaired generation of hepatitis $B$ virus-specific memory B cells in HIV infected individuals following vaccination. Vaccine. 2010; 28(21):3672-3678.

43. Herbeuval JP, et al. Differential expression of IFN-alpha and TRAIL/DR5 in lymphoid tissue of progressor versus nonprogressor HIV-1infected patients. Proc Natl Acad Sci U S A. 2006; 103(18):7000-7005.

44. Herbeuval JP, Shearer GM. HIV-1 immunopathogenesis: how good interferon turns bad. Clin Immunol. 2007;123(2):121-128.

45. Stahl M, et al. The forkhead transcription factor FoxO regulates transcription of $\mathrm{p} 27 \mathrm{Kip} 1$ and $\mathrm{Bim}$ in response to IL-2. I Immunol. 2002 168(10):5024-5031.

46. Breen EC. Pro- and anti-inflammatory cytokines in human immunodeficiency virus infection and acquired immunodeficiency syndrome. Pharmacol Ther. 2002;95(3):295-304

47. Decrion AZ, Dichamp I, Varin A, Herbein G. HIV and inflammation. Curr HIV Res. 2005;3(3):243-259.

48. Vicenzi E, Biswas P, Mengozzi M, Poli G. Role of pro-inflammatory cytokines and beta-chemokines in controlling HIV replication. J Leukoc Biol. 1997;62(1):34-40

49. Estes JD, et al. Simian immunodeficiency virusinduced lymphatic tissue fibrosis is mediated by transforming growth factor beta 1-positive regulatory $\mathrm{T}$ cells and begins in early infection. J Infect Dis. 2007;195(4):551-561

50. Brenchley JM, et al. Microbial translocation is a cause of systemic immune activation in chronic HIV infection. Nat Med. 2006;12(12):1365-1371.
51. Parry RV, et al. CTLA-4 and PD-1 receptors inhibit T-cell activation by distinct mechanisms. Mol Cell Biol. 2005;25(21):9543-9553.

52. Titanji K, et al. Acute depletion of activated memory B cells involves the PD-1 pathway in rapidly progressing SIV-infected macaques. $J$ Clin Invest. 2010;120(11):3878-3890.

53. Roy MP, Kim CH, Butcher EC. Cytokine control of memory B cell homing machinery. J Immunol. 2002;169(4):1676-1682.

54 . Beniguel L, et al. Specific antibody production by blood B cells is retained in late stage drug-naive HIV-infected Africans. Clin Dev Immunol. 2004; 11(2):121-127.

55. Pinna D, Corti D, Jarrossay D, Sallusto F, Lanzavecchia A. Clonal dissection of the human memory Bcell repertoire following infection and vaccination. Eur J Immunol. 2009;39(5):1260-1270.

56. Levy Y, et al. Effects of interleukin-2 therapy combined with highly active antiretroviral therapy on immune restoration in HIV-1 infection: a randomized controlled trial. Aids. 2003;17(3):343-351.

57. Moir S, et al. B cells in early and chronic HIV infection: evidence for preservation of immune function associated with early initiation of antiretroviral therapy. Blood. 2010;116(25):5571-5579.

58. Schacker TW, et al. Lymphatic tissue fibrosis is associated with reduced numbers of naive $\mathrm{CD} 4+\mathrm{T}$ cells in human immunodeficiency virus type 1 infection. Clin Vaccine Immunol. 2006;13(5):556-560.

59. Cretney E, Shanker A, Yagita H, Smyth MJ, Sayers TJ. TNF-related apoptosis-inducing ligand as a therapeutic agent in autoimmunity and cancer. Immunol Cell Biol. 2006;84(1):87-98.

60. Essafi M, Baudot AD, Mouska X, Cassuto JP, Ticchioni M, Deckert M. Cell-penetrating TAT-FOXO3 fusion proteins induce apoptotic cell death in leukemic cells. Mol Cancer Ther. 2011;10(1):37-46.

61. Kikuchi S, Nagai T, Kunitama M, Kirito K, Ozawa K, Komatsu N. Active FKHRL1 overcomes imatinib resistance in chronic myelogenous leukemia-derived cell lines via the production of tumor necrosis factor-related apoptosis-inducing ligand. Cancer Sci. 2007;98(12):1949-1958. 